\title{
On Solutions of Variational Inequality Problems via Iterative Methods
}

\author{
Mohammed Ali Alghamdi, ${ }^{1}$ Naseer Shahzad, ${ }^{1}$ and Habtu Zegeye ${ }^{2}$ \\ ${ }^{1}$ Department of Mathematics, King Abdulaziz University, P.O. Box 80203, Jeddah 21589, Saudi Arabia \\ ${ }^{2}$ Department of Mathematics, University of Botswana, Private Bag 00704, Gaborone, Botswana
}

Correspondence should be addressed to Naseer Shahzad; nshahzad@kau.edu.sa

Received 12 May 2014; Revised 24 June 2014; Accepted 30 June 2014; Published 4 August 2014

Academic Editor: Adrian Petrusel

Copyright (C) 2014 Mohammed Ali Alghamdi et al. This is an open access article distributed under the Creative Commons Attribution License, which permits unrestricted use, distribution, and reproduction in any medium, provided the original work is properly cited.

We investigate an algorithm for a common point of fixed points of a finite family of Lipschitz pseudocontractive mappings and solutions of a finite family of $\gamma$-inverse strongly accretive mappings. Our theorems improve and unify most of the results that have been proved in this direction for this important class of nonlinear mappings.

\section{Introduction}

Let $C$ be a subset of a real Hilbert space $H$. Let $A: C \rightarrow H$ be a nonlinear mapping. The variational inequality problem for $A$ and $C$ is to

find $x^{*} \in C$ such that $\left\langle A x^{*}, v-x^{*}\right\rangle \geq 0, \quad \forall v \in C$.

The set of solutions of variational inequality problem is denoted by $\operatorname{VI}(C, A)$; that is,

$$
\mathrm{VI}(C, A)=\left\{x^{*} \in C:\left\langle A x^{*}, x-x^{*}\right\rangle \geq 0, \forall x \in C\right\} .
$$

It is well known that variational inequality theory has emerged as an important tool in studying a wide class of numerous problems in variational inequalities, minimax problems, optimization, physics, and the Nash equilibrium problems in noncooperative games. Several numerical methods have been developed for solving variational inequalities and related optimization problems; see, for instance, [1-5] and the references therein.

A mapping $A: C \subseteq H \rightarrow H$ is said to be $\gamma$-inverse strongly accretive (or $\gamma$-inverse strongly monotone) if there exists a positive real number $\gamma$ such that

$$
\langle x-y, A x-A y\rangle \geq \gamma\|A x-A y\|^{2}, \quad \forall x, y \in C .
$$

If $A$ is $\gamma$-inverse strongly accretive, then inequality (3) implies that $A$ is Lipschitzian with constant $L:=1 / \gamma$; that is, $\|A x-A y\| \leq(1 / \gamma)\|x-y\|$, for all $x, y \in C$. If in (3) we have that $\gamma=0$, then $A$ is called accretive (or monotone).

Let $C$ be a closed and convex subset of a real Hilbert space $H$. A mapping $T: C \rightarrow H$ is called a contraction mapping if there exists $L \in[0,1)$ such that $\|T x-T y\| \leq L\|x-y\|$ for all $x, y \in C$. If $L=1$, then $T$ is called nonexpansive. A mapping $T: C \rightarrow E$ is called $\lambda$-strictly pseudocontractive of BrowderPetryshyn type [6] if and only if there exists $\lambda \in(0,1)$ such that

$$
\begin{array}{r}
\|T x-T y\|^{2} \leq\|x-y\|^{2}+\lambda\|(I-T) x-(I-T) y\|^{2} \\
\forall x, y \in C .
\end{array}
$$

$T$ is called pseudocontractive if

$$
\begin{array}{r}
\|T x-T y\|^{2} \leq\|x-y\|^{2}+\|(I-T) x-(I-T) y\|^{2}, \\
\forall x, y \in C .
\end{array}
$$

We note that inequalities (4) and (5) can be equivalently written as

$$
\begin{array}{r}
\langle T x-T y, x-y\rangle \leq\|x-y\|^{2}-k\|(x-T x)-(y-T y)\|^{2} \\
\forall x, y \in C,
\end{array}
$$


for some $k>0$ and

$$
\langle T x-T y, x-y\rangle \leq\|x-y\|^{2} \quad \forall x, y \in C,
$$

respectively. We remark that $T$ is pseudocontractive if and only if $A:=(I-T)$ is accretive. A point $x \in C$ is a fixed point of $T$ if $T x=x$ and we denote by $F(T)$ the set of fixed points of $T$; that is, $F(T)=\{x \in C: T x=x\}$.

We observe that in a real Hilbert space $H$ a class of pseudocontractive mappings includes the class of $\lambda$-strictly pseudocontractive mappings and hence the classes of nonexpansive and contraction mappings.

Closely related to the variational inequality problems is the problem of finding fixed points of nonexpansive mappings, $\lambda$-strict pseudocontraction mappings or pseudocontractive mappings which is the current interest in functional analysis. Several researchers considered a unified approach that approximates a common point of fixed point of nonlinear problems and solutions of variational inequality problems and solutions of variational inequality problems; see, for example, [7-18] and the references therein.

In [19], Takahashi and Toyoda studied the problem of finding a common point of fixed points of a nonexpansive mapping and solutions of a variational inequality problem (1) by considering the following iterative algorithm:

$$
\begin{gathered}
x_{0} \in C, \\
x_{n+1}=\alpha_{n} x_{n}+\left(1-\alpha_{n}\right) T P_{C}\left(x_{n}-\lambda_{n} A x_{n}\right), \quad n=0,1, \ldots,
\end{gathered}
$$

where $\left\{\alpha_{n}\right\}$ is a sequence in $(0,1),\left\{\lambda_{n}\right\}$ is a positive sequence, $T: C \rightarrow C$ is a nonexpansive mapping, and $A: C \rightarrow H$ is an $\gamma$-inverse strongly accretive mapping. They showed that the sequence $\left\{x_{n}\right\}$ generated by (8) converges weakly to some $z \in$ $\mathrm{VI}(C, A) \cap F(S)$ provided that the control sequences satisfy some restrictions.

Iiduka and Takahashi [20] reconsidered the common element problem via the following iterative algorithm:

$$
\begin{gathered}
x_{1}=x \in C, \\
x_{n+1}=\alpha_{n} x+\left(1-\alpha_{n}\right) T P_{C}\left(x_{n}-\lambda_{n} A x_{n}\right), \quad n=0,1, \ldots,
\end{gathered}
$$

where $T: C \rightarrow C$ is a nonexpansive mapping, $A: C \rightarrow H$ is a $\gamma$-inverse-strongly accretive mapping, $\left\{\alpha_{n}\right\}$ is a sequence in $(0,1)$, and $\left\{\lambda_{n}\right\}$ is a sequence in $(0,2 \alpha)$. They proved that the sequence $\left\{x_{n}\right\}$ strongly converges to some point $z \in F(T) \cap$ $\mathrm{VI}(C, A)$.

Recently, Zegeye and Shahzad [21] investigated the problem of finding a common point of fixed points of a Lipschitz pseudocontractive mapping $T$ and solutions of a variational inequality problem for $\gamma$-inverse strongly accretive mapping $A$ by considering the following iterative algorithm:

$$
\begin{gathered}
y_{n}=\left(1-\beta_{n}\right) x_{n}+\beta_{n} T x_{n} \\
x_{n+1}=P_{C}\left[\left(1-\alpha_{n}\right)\left(\delta_{n} T y_{n}+\theta_{n} x_{n}+\gamma_{n} P_{C}[I-\gamma A] x_{n}\right)\right],
\end{gathered}
$$

where $P_{C}$ is a metric projection from $H$ onto $C$ and $\left\{\delta_{n}\right\},\left\{\theta_{n}\right\},\left\{\gamma_{n}\right\},\left\{\alpha_{n}\right\},\left\{\beta_{n}\right\}$ are in $(0,1)$ satisfying certain conditions. Then, they proved that the sequence $\left\{x_{n}\right\}$ converges strongly to the minimum-norm point of $F(T) \cap$ $\mathrm{VI}(C, A)$.

A natural question arises whether we can obtain an iterative scheme which converges strongly to a common point of fixed points of a finite family of pseudocontractive mappings and solutions of a finite family of variational inequality problems for $\gamma$-inverse strongly accretive mappings or not.

It is our purpose in this paper to introduce an algorithm and prove that the algorithm converges strongly to a common point of fixed points of a finite family of Lipschitz pseudocontractive mappings and solutions of a finite family of variational inequality problems for $\gamma$-inverse strongly accretive mappings. The results obtained in this paper improve and extend the results of Takahashi and Toyoda [19], Iiduka and Takahashi [20], and Zegeye and Shahzad [21], Theorem 3.2 of Yao et al. [22], and some other results in this direction.

\section{Preliminaries}

In what follows we will make use of the following lemmas.

Lemma 1. Letting $H$ be a real Hilbert space, the following identity holds:

$$
\|x+y\|^{2} \leq\|x\|^{2}+2\langle y, x+y\rangle, \quad \forall x, y \in H .
$$

Lemma 2 (see [23]). Let $C$ be a nonempty closed and convex subset of a real Hilbert space $H$. Let $A: C \rightarrow E$ be a $\gamma$-inverse strongly accretive mapping. Then, for $0<\mu<2 \gamma$, the mapping $A_{\mu} x:=(x-\mu A x)$ is nonexpansive.

Lemma 3 (see [24]). Let $C$ be a nonempty, closed, and convex subset of a smooth Banach space E. Let $Q_{C}$ be a sunny nonexpansive retraction from $E$ onto $C$ and let $A$ be an accretive operator of $C$ into $E$. Then for all $\lambda>0$,

$$
V I(C, A)=F\left(Q_{C}(I-\lambda A)\right) \text {. }
$$

Lemma 4 (see [25]). Let $C$ be a nonempty, closed, and convex subset of a real Hilbert space $H$. Let $T_{i}: C \rightarrow E, i=1, \ldots, N$, be nonexpansive mappings such that $\cap_{i=1}^{N} F\left(T_{i}\right) \neq \emptyset$. Let $T:=$ $\theta_{1} T_{1}+\theta_{2} T_{2}+\cdots+\theta_{N} T_{N}$ with $\theta_{1}+\theta_{2}+\cdots+\theta_{N}=1$. Then $T$ is nonexpansive and $F(T)=\cap_{i=1}^{N} F\left(T_{i}\right)$.

Lemma 5 (see [26]). Let $C$ be a convex subset of a real Hilbert space $H$. Let $x \in H$. Then $x_{0}=P_{C} x$ if and only if

$$
\left\langle z-x_{0}, x-x_{0}\right\rangle \leq 0, \quad \forall z \in C .
$$

Lemma 6 (see [27]). Let $C$ be a closed convex subset of a real Hilbert space $H$ and $A: C \rightarrow C$ be a continuous pseudocontractive mapping. Then, for $0<\mu<2 \gamma$, the mapping $A_{\mu} x:=(x-\mu A x)$ is nonexpansive

(i) $F(T)$ is a closed convex subset of $C$;

(ii) $(I-T)$ is demiclosed at zero; that is, if $\left\{x_{n}\right\}$ is a sequence in $C$ such that $x_{n} \rightarrow x$ and $T x_{n}-x_{n} \rightarrow 0$, as $n \rightarrow \infty$, then $x=T(x)$. 
Lemma 7 (see [28]). Let $H$ be a real Hilbert space. Then for all $x_{i} \in H$ and $\alpha_{i} \in[0,1]$ for $i=1,2,3$ such that $\alpha_{1}+\alpha_{2}+\alpha_{3}=1$ the following equality holds:

$$
\left\|\alpha_{1} x_{1}+\alpha_{2} x_{2}+\alpha_{3} x_{3}\right\|^{2}=\sum_{i=1}^{3} \alpha_{i}\left\|x_{i}\right\|^{2}-\sum_{1 \leq i, j \leq 3} \alpha_{i} \alpha_{j}\left\|x_{i}-x_{j}\right\|^{2} .
$$

Lemma 8 (see [29]). Let $\left\{a_{n}\right\}$ be sequences of real numbers such that there exists a subsequence $\left\{n_{i}\right\}$ of $\{n\}$ such that $a_{n_{i}}<$ $a_{n_{i}+1}$ for all $i \in \mathbb{N}$. Then there exists an increasing sequence $\left\{m_{k}\right\} \subset \mathbb{N}$ such that $m_{k} \rightarrow \infty$ and the following properties are satisfied by all (sufficiently large) numbers $k \in \mathbb{N}$ :

$$
a_{m_{k}} \leq a_{m_{k}+1}, \quad a_{k} \leq a_{m_{k}+1}
$$

In fact, $m_{k}$ is the largest number $n$ in the set $\{1,2, \ldots, k\}$ such that the condition $a_{n} \leq a_{n+1}$ holds.

Lemma 9 (see [30]). Let $\left\{a_{n}\right\}$ be a sequence of nonnegative real numbers satisfying the following relation:

$$
a_{n+1} \leq\left(1-\alpha_{n}\right) a_{n}+\alpha_{n} \delta_{n}, \quad n \geq n_{0},
$$

where $\left\{\alpha_{n}\right\} \subset(0,1)$ and $\left\{\delta_{n}\right\} \subset \mathbb{R}$ satisfying the following conditions: $\lim _{n \rightarrow \infty} \alpha_{n}=0, \sum_{n=1}^{\infty} \alpha_{n}=\infty$ and $\lim \sup _{n \rightarrow \infty} \delta_{n} \leq 0$. Then, $\lim _{n \rightarrow \infty} a_{n}=0$.

\section{Main Result}

For the rest of this paper, let $\left\{a_{n}\right\},\left\{b_{n}\right\},\left\{c_{n}\right\}, \subset(c, 1) \subset(0,1)$, for some $c \in(0,1)$, and $\left\{\alpha_{n}\right\} \subset(0, b) \subset(0,1)$, for some $b \in$ $(0,1)$, satisfy (i) $a_{n}+b_{n}+c_{n}=1$; (ii) $\lim _{n \rightarrow \infty} \alpha_{n}=0$; and (iii) $\sum \alpha_{n}=\infty$.

Theorem 10. Let $C$ be a nonempty, closed, and convex subset of a real Hilbert space $H$. Let $T_{j}: C \rightarrow C, j=1,2, \ldots, M$, be Lipschitz pseudocontractive mappings with Lipschitz constants $L_{i}$, respectively. Let $A_{j}: C \rightarrow H$, for $j=1,2, \ldots, N$, be $\gamma_{j}$-inverse strongly accretive mappings. Let $f: C \rightarrow$ $C$ be a contraction with constant $\alpha$. Assume that $\mathscr{F}=$ $\left[\cap_{j=1}^{M} F\left(T_{j}\right)\right] \bigcap\left[\cap_{j=1}^{N} V I\left(C, A_{j}\right)\right]$ is nonempty. Let a sequence $\left\{x_{n}\right\}$ be generated from an arbitrary $x_{0} \in C$ by

$$
\begin{gathered}
y_{n}=\left(1-\lambda_{n}\right) x_{n}+\lambda_{n} T_{n} x_{n} \\
x_{n+1}=\alpha_{n} f\left(x_{n}\right)+\left(1-\alpha_{n}\right)\left(a_{n} x_{n}+b_{n} T_{n} y_{n}+c_{n} G x_{n}\right),
\end{gathered}
$$

where $T_{n}=T_{n(\bmod M)}$ and $G:=e_{0} I+e_{1} P_{C}\left[I-\gamma A_{1}\right]+$ $e_{2} P_{C}\left[I-\gamma A_{2}\right]+\cdots+e_{N} P_{C}\left[I-\gamma A_{r}\right]$, for $\gamma \in\left(0,2 \gamma_{0}\right)$, for $\gamma_{0}:=$ $\min _{1 \leq j \leq N}\left\{\gamma_{j}\right\}$ with $e_{0}+e_{1}+\cdots+e_{r}=1$ and $b_{n}+c_{n} \leq \lambda_{n} \leq \lambda<$ $1 /\left(\sqrt{1+L^{2}}+1\right), \forall n \geq 0$, for $L=\max \left\{L_{j}: 1 \leq j \leq M\right\}$. Then, $\left\{x_{n}\right\}$ converges strongly to a point $x^{*} \in \mathscr{F}$ which is the unique solution of the variational inequality $\left\langle(I-f)\left(x^{*}\right), x-x^{*}\right\rangle \geq 0$ for all $x \in \mathscr{F}$.
Proof. From Lemmas 2, 4, and 3 we get that $G$ is nonexpansive mapping with $F(G)=\cap_{j=1}^{N} \operatorname{VI}\left(C, A_{j}\right)$. Let $p \in \mathscr{F}$. Then from (17), (5), and Lemma 7 we have that

$$
\begin{aligned}
& \left\|y_{n}-p\right\|^{2} \\
& =\left\|\left(1-\lambda_{n}\right)\left(x_{n}-p\right)+\lambda_{n}\left(T_{n} x_{n}-p\right)\right\|^{2} \\
& =\left(1-\lambda_{n}\right)\left\|x_{n}-p\right\|^{2}+\lambda_{n}\left\|T_{n} x_{n}-p\right\|^{2} \\
& -\lambda_{n}\left(1-\lambda_{n}\right)\left\|x_{n}-T_{n} x_{n}\right\|^{2} \\
& \leq\left(1-\lambda_{n}\right)\left\|x_{n}-p\right\|^{2} \\
& +\lambda_{n}\left[\left\|x_{n}-p\right\|^{2}+\left\|x_{n}-T_{n} x_{n}\right\|^{2}\right] \\
& -\lambda_{n}\left(1-\lambda_{n}\right)\left\|x_{n}-T_{n} x_{n}\right\|^{2} \\
& =\left\|x_{n}-p\right\|^{2}+\lambda_{n}^{2}\left\|x_{n}-T_{n} x_{n}\right\|^{2}, \\
& \left\|x_{n+1}-p\right\|^{2} \\
& =\left\|\alpha_{n} f\left(x_{n}\right)+\left(1-\alpha_{n}\right)\left(a_{n} x_{n}+b_{n} T_{n} y_{n}+c_{n} G x_{n}\right)-p\right\|^{2} \\
& =\| \alpha_{n}\left(f\left(x_{n}\right)-p\right)+\left(1-\alpha_{n}\right) \\
& \times\left(a_{n}\left(x_{n}-p\right)+b_{n}\left(T_{n} y_{n}-p\right)+c_{n}\left(G x_{n}-p\right)\right) \|^{2} \\
& \leq \alpha_{n}\left\|f\left(x_{n}\right)-p\right\|^{2}+\left(1-\alpha_{n}\right) \\
& \times\left\|a_{n}\left(x_{n}-p\right)+b_{n}\left(T_{n} y_{n}-p\right)+c_{n}\left(G x_{n}-p\right)\right\|^{2} \\
& \leq \alpha_{n}\left\|f\left(x_{n}\right)-p\right\|^{2}+\left(1-\alpha_{n}\right) \\
& \times\left[a_{n}\left\|x_{n}-p\right\|^{2}+b_{n}\left\|T_{n} y_{n}-p\right\|^{2}\right. \\
& \left.+c_{n}\left\|G x_{n}-p\right\|^{2}\right]-\left(1-\alpha_{n}\right) b_{n} a_{n}\left\|T_{n} y_{n}-x_{n}\right\|^{2} \\
& \leq \alpha_{n}\left\|f\left(x_{n}\right)-p\right\|^{2}+\left(1-\alpha_{n}\right)\left[\left(a_{n}+c_{n}\right)\left\|x_{n}-p\right\|^{2}\right. \\
& \left.+b_{n}\left\|T_{n} y_{n}-p\right\|^{2}\right] \\
& -\left(1-\alpha_{n}\right) b_{n} a_{n}\left\|T_{n} y_{n}-x_{n}\right\|^{2} \\
& \leq \alpha_{n}\left\|f\left(x_{n}\right)-p\right\|^{2} \\
& +\left(1-\alpha_{n}\right)\left[\left(a_{n}+c_{n}\right)\left\|x_{n}-p\right\|^{2}\right. \\
& \left.+b_{n}\left(\left\|y_{n}-p\right\|^{2}+\left\|y_{n}-T_{n} y_{n}\right\|^{2}\right)\right] \\
& -\left(1-\alpha_{n}\right) b_{n} a_{n}\left\|T_{n} y_{n}-x_{n}\right\|^{2} \text {. }
\end{aligned}
$$

Now, substituting (18) in (19) we get that

$$
\begin{aligned}
& \left\|x_{n+1}-p\right\|^{2} \\
& \leq \alpha_{n}\left\|f\left(x_{n}\right)-p\right\|^{2} \\
& +\left(1-\alpha_{n}\right)\left[\left(a_{n}+c_{n}\right)\left\|x_{n}-p\right\|^{2}\right. \\
& +b_{n}\left(\left\|x_{n}-p\right\|^{2}+\lambda_{n}^{2}\left\|x_{n}-T_{n} x_{n}\right\|^{2}\right) \\
& \left.+b_{n}\left\|y_{n}-T_{n} y_{n}\right\|^{2}\right] \\
& -\left(1-\alpha_{n}\right) b_{n} a_{n}\left\|T_{n} y_{n}-x_{n}\right\|^{2}
\end{aligned}
$$




$$
\begin{aligned}
= & \alpha_{n}\left\|f\left(x_{n}\right)-p\right\|^{2}+\left(1-\alpha_{n}\right)\left\|x_{n}-p\right\|^{2} \\
& +\left(1-\alpha_{n}\right) \lambda_{n}^{2} b_{n}\left\|x_{n}-T_{n} x_{n}\right\|^{2} \\
& +\left(1-\alpha_{n}\right) b_{n}\left\|y_{n}-T_{n} y_{n}\right\|^{2} \\
& -\left(1-\alpha_{n}\right) b_{n} a_{n}\left\|T_{n} y_{n}-x_{n}\right\|^{2} .
\end{aligned}
$$

Moreover, from (17), Lemma 7, and Lipschitz property of $T_{n}$ we get that

$$
\begin{aligned}
\| y_{n}- & T_{n} y_{n} \|^{2} \\
= & \left\|\left(1-\lambda_{n}\right)\left(x_{n}-T_{n} y_{n}\right)+\lambda_{n}\left(T_{n} x_{n}-T_{n} y_{n}\right)\right\|^{2} \\
= & \left(1-\lambda_{n}\right)\left\|x_{n}-T_{n} y_{n}\right\|^{2}+\lambda_{n}\left\|T_{n} x_{n}-T_{n} y_{n}\right\|^{2} \\
& -\lambda_{n}\left(1-\lambda_{n}\right)\left\|x_{n}-T_{n} x_{n}\right\|^{2} \\
\leq & \left(1-\lambda_{n}\right)\left\|x_{n}-T_{n} y_{n}\right\|^{2}+\lambda_{n} L^{2}\left\|x_{n}-y_{n}\right\|^{2} \\
& -\lambda_{n}\left(1-\lambda_{n}\right)\left\|x_{n}-T_{n} x_{n}\right\|^{2} \\
= & \left(1-\lambda_{n}\right)\left\|x_{n}-T_{n} y_{n}\right\|^{2}+\lambda_{n}^{3} L^{2}\left\|x_{n}-T_{n} x_{n}\right\|^{2} \\
& -\lambda_{n}\left(1-\lambda_{n}\right)\left\|x_{n}-T_{n} x_{n}\right\|^{2} \\
= & \left(1-\lambda_{n}\right)\left\|x_{n}-T_{n} y_{n}\right\|^{2} \\
& -\lambda_{n}\left(1-L^{2} \lambda_{n}^{2}-\lambda_{n}\right)\left\|x_{n}-T_{n} x_{n}\right\|^{2} .
\end{aligned}
$$

Substituting (21) into (20) we obtain that

$$
\begin{aligned}
\left\|x_{n+1}-p\right\|^{2} & \\
\leq & \alpha_{n}\left\|f\left(x_{n}\right)-p\right\|^{2}+\left(1-\alpha_{n}\right)\left\|x_{n}-p\right\|^{2} \\
& +\left(1-\alpha_{n}\right) b_{n} \lambda_{n}^{2}\left\|x_{n}-T_{n} x_{n}\right\|^{2} \\
& +\left(1-\alpha_{n}\right) b_{n}\left[\left(1-\lambda_{n}\right)\left\|x_{n}-T_{n} y_{n}\right\|^{2}\right. \\
& \left.-\lambda_{n}\left(1-L^{2} \lambda_{n}^{2}-\lambda_{n}\right)\left\|x_{n}-T_{n} x_{n}\right\|^{2}\right] \\
& -\left(1-\alpha_{n}\right) b_{n} a_{n}\left\|T_{n} y_{n}-x_{n}\right\|^{2}, \\
= & \alpha_{n}\left\|f\left(x_{n}\right)-p\right\|^{2}+\left(1-\alpha_{n}\right)\left\|x_{n}-p\right\|^{2} \\
& -\left(1-\alpha_{n}\right) \lambda_{n} b_{n}\left[1-L^{2} \lambda_{n}^{2}-2 \lambda_{n}\right]\left\|x_{n}-T_{n} x_{n}\right\|^{2} \\
& +\left(1-\alpha_{n}\right) b_{n}\left[\left(1-a_{n}\right)-\lambda_{n}\right]\left\|T_{n} y_{n}-x_{n}\right\|^{2} \\
= & \alpha_{n}\left\|f\left(x_{n}\right)-p\right\|^{2}+\left(1-\alpha_{n}\right)\left\|x_{n}-p\right\|^{2} \\
& -\left(1-\alpha_{n}\right) \lambda_{n} b_{n}\left[1-L^{2} \lambda_{n}^{2}-2 \lambda_{n}\right]\left\|x_{n}-T_{n} x_{n}\right\|^{2} \\
& +\left(1-\alpha_{n}\right) b_{n}\left[b_{n}+c_{n}-\lambda_{n}\right]\left\|T_{n} y_{n}-x_{n}\right\|^{2} .
\end{aligned}
$$

But, from the hypothesis we have that

$$
\begin{gathered}
1-2 \lambda_{n}-L^{2} \lambda_{n}^{2} \geq 1-2 \lambda-L^{2} \lambda^{2}>0, \\
b_{n}+c_{n} \leq \lambda_{n}, \quad \forall n \geq 0,
\end{gathered}
$$

and hence inequality (22) gives that

$$
\left\|x_{n+1}-p\right\|^{2} \leq \alpha_{n}\left\|f\left(x_{n}\right)-p\right\|^{2}+\left(1-\alpha_{n}\right)\left\|x_{n}-p\right\|^{2} \text {. }
$$

But we have that

$$
\begin{aligned}
\| f & \left(x_{n}\right)-p \|^{2} \\
& =\left[\left\|f\left(x_{n}\right)-f(p)\right\|+\|f(p)-p\|\right]^{2} \\
& \leq\left[\alpha\left\|x_{n}-p\right\|+\|f(p)-p\|\right]^{2} \\
& \leq \alpha^{2}\left\|x_{n}-p\right\|^{2}+\|f(p)-p\|^{2}+2 \alpha\left\|x_{n}-p\right\|\|f(p)-p\| \\
& \leq \alpha(1+\alpha)\left\|x_{n}-p\right\|^{2}+(1+\alpha)\|f(p)-p\|^{2} .
\end{aligned}
$$

Substituting (25) into (24) we get that

$$
\begin{aligned}
& \left\|x_{n+1}-p\right\|^{2} \\
& \quad \leq\left(1-\alpha_{n}(1-\alpha(1+\alpha))\right)\left\|x_{n}-p\right\|^{2} \\
& \quad+\alpha_{n}(1+\alpha)\|f(p)-p\|^{2} .
\end{aligned}
$$

Therefore, by induction we get that

$$
\begin{aligned}
& \left\|x_{n+1}-p\right\|^{2} \\
& \quad \leq \max \left\{\left\|x_{0}-p\right\|^{2}, \frac{1+\alpha}{1-\alpha(1+\alpha)}\|f(p)-p\|^{2}\right\},
\end{aligned}
$$

$\forall n \geq 0$, 
Let $x^{*}=P_{\mathscr{F}} f\left(x^{*}\right)$. Then, from (17), Lemmas 1 and 7, and the methods used to get (22) we obtain that

$$
\begin{aligned}
& \left\|x_{n+1}-x^{*}\right\|^{2} \\
& =\| \alpha_{n}\left(f\left(x_{n}\right)-x^{*}\right) \\
& +\left(1-\alpha_{n}\right)\left[a_{n} x_{n}+b_{n} T_{n} y_{n}+c_{n} G x_{n}-x^{*}\right] \|^{2} \\
& \leq\left(1-\alpha_{n}\right) \| a_{n}\left(x_{n}-x^{*}\right)+b_{n}\left(T_{n} y_{n}-x^{*}\right) \\
& +c_{n}\left(G x_{n}-x^{*}\right) \|^{2} \\
& +2 \alpha_{n}\left\langle f\left(x_{n}\right)-x^{*}, x_{n+1}-x^{*}\right\rangle \\
& \leq\left(1-\alpha_{n}\right) b_{n}\left\|T_{n} y_{n}-x^{*}\right\|^{2}+\left(1-\alpha_{n}\right) a_{n}\left\|x_{n}-x^{*}\right\|^{2} \\
& \times\left(1-\alpha_{n}\right) c_{n}\left\|G x_{n}-p\right\|^{2} \\
& -\left(1-\alpha_{n}\right) a_{n} b_{n}\left\|T_{n} y_{n}-x_{n}\right\|^{2} \\
& -\left(1-\alpha_{n}\right) a_{n} c_{n}\left\|G x_{n}-x_{n}\right\|^{2} \\
& +2 \alpha_{n}\left\langle f\left(x_{n}\right)-x^{*}, x_{n+1}-x^{*}\right\rangle \text {, } \\
& \left\|x_{n+1}-x^{*}\right\|^{2} \\
& \leq\left(1-\alpha_{n}\right) b_{n}\left[\left\|y_{n}-x^{*}\right\|^{2}+\left\|y_{n}-T_{n} y_{n}\right\|^{2}\right] \\
& +\left(1-\alpha_{n}\right)\left(a_{n}+c_{n}\right)\left\|x_{n}-x^{*}\right\|^{2} \\
& -\left(1-\alpha_{n}\right) a_{n} b_{n}\left\|T_{n} y_{n}-x_{n}\right\|^{2} \\
& -\left(1-\alpha_{n}\right) a_{n} c_{n}\left\|G x_{n}-x_{n}\right\|^{2} \\
& +2 \alpha_{n}\left\langle f\left(x_{n}\right)-x^{*}, x_{n+1}-x^{*}\right\rangle \text {, } \\
& \leq\left(1-\alpha_{n}\right) b_{n}\left[\left\|x_{n}-x^{*}\right\|^{2}+\lambda_{n}^{2}\left\|x_{n}-T_{n} x_{n}\right\|^{2}\right] \\
& +\left(1-\alpha_{n}\right) b_{n}\left[\left(1-\lambda_{n}\right)\left\|x_{n}-T_{n} y_{n}\right\|^{2}\right. \\
& -\lambda_{n}\left(1-L^{2} \lambda_{n}^{2}-\lambda_{n}\right) \\
& \left.\times\left\|x_{n}-T_{n} x_{n}\right\|^{2}\right] \\
& +\left(1-\alpha_{n}\right)\left(a_{n}+c_{n}\right)\left\|x_{n}-x^{*}\right\|^{2} \\
& -\left(1-\alpha_{n}\right) a_{n} b_{n}\left\|T_{n} y_{n}-x_{n}\right\|^{2} \\
& -\left(1-\alpha_{n}\right) a_{n} c_{n}\left\|G x_{n}-x_{n}\right\|^{2} \\
& +2 \alpha_{n}\left\langle f\left(x_{n}\right)-x^{*}, x_{n+1}-x^{*}\right\rangle
\end{aligned}
$$

which implies that

$$
\begin{aligned}
\| x_{n+1} & -x^{*} \|^{2} \\
\leq & \left(1-\alpha_{n}\right)\left\|x_{n}-x^{*}\right\|^{2}-\left(1-\alpha_{n}\right) b_{n} \lambda_{n}\left[1-L^{2} \lambda_{n}^{2}-2 \lambda_{n}\right] \\
& \times\left\|x_{n}-T_{n} x_{n}\right\|^{2}+\left(1-\alpha_{n}\right) b_{n}\left(b_{n}+c_{n}-\lambda_{n}\right)\left\|x_{n}-T_{n} y_{n}\right\|^{2} \\
& -\left(1-\alpha_{n}\right) a_{n} d_{n}\left\|G x_{n}-x_{n}\right\|^{2} \\
& +2 \alpha_{n}\left\langle f\left(x_{n}\right)-x^{*}, x_{n+1}-x^{*}\right\rangle
\end{aligned}
$$

$$
\leq\left(1-\alpha_{n}\right)\left\|x_{n}-x^{*}\right\|^{2}+2 \alpha_{n}\left\langle f\left(x_{n}\right)-x^{*}, x_{n+1}-x^{*}\right\rangle .
$$

But

$$
\begin{aligned}
\langle f & \left.\left(x_{n}\right)-x^{*}, x_{n+1}-x^{*}\right\rangle \\
= & \left\langle f\left(x_{n}\right)-x^{*}, x_{n}-x^{*}\right\rangle+\left\langle f\left(x_{n}\right)-x^{*}, x_{n+1}-x_{n}\right\rangle \\
\leq & \left\langle f\left(x_{n}\right)-f\left(x^{*}\right), x_{n}-x^{*}\right\rangle+\left\langle f\left(x^{*}\right)-x^{*}, x_{n}-x^{*}\right\rangle \\
& +\left\|x_{n+1}-x_{n}\right\|\left\|f\left(x_{n}\right)-x^{*}\right\| \\
\leq & \alpha\left\|x_{n}-x^{*}\right\|^{2}+\left\langle f\left(x^{*}\right)-x^{*}, x_{n}-x^{*}\right\rangle \\
& +\left\|x_{n+1}-x_{n}\right\|\left\|f\left(x_{n}\right)-x^{*}\right\| .
\end{aligned}
$$

Thus, substituting (31) in (30) we obtain that

$$
\begin{aligned}
\| x_{n+1} & -x^{*} \|^{2} \\
\leq & \left(1-\alpha_{n}(1-2 \alpha)\right)\left\|x_{n}-x^{*}\right\|^{2} \\
& +2 \alpha_{n}\left\langle f\left(x^{*}\right)-x^{*}, x_{n}-x^{*}\right\rangle \\
& +2 \alpha_{n}\left\|x_{n+1}-x_{n}\right\| \cdot\left\|f\left(x_{n}\right)-x^{*}\right\| .
\end{aligned}
$$

Next, we consider two cases.

Case 1. Suppose that there exists $n_{0} \in \mathbb{N}$ such that $\left\{\left\|x_{n}-x^{*}\right\|\right\}$ is decreasing for all $n \geq n_{0}$. Then, we get that $\left\{\left\|x_{n}-x^{*}\right\|\right\}$ is convergent. Thus, from (29) and (23) we have that

$$
x_{n}-T_{n} x_{n} \longrightarrow 0, \quad G x_{n}-x_{n} \longrightarrow 0 \quad \text { as } n \longrightarrow \infty \text {. }
$$

Furthermore, from (17) and (33) we obtain that

$$
\left\|y_{n}-x_{n}\right\|=\lambda_{n}\left\|x_{n}-T_{n} x_{n}\right\| \longrightarrow 0 \text { as } n \longrightarrow \infty,
$$

and hence Lipschitz continuity of $T_{n}$, (34), and (33) implies that

$$
\begin{aligned}
& \left\|T_{n} y_{n}-x_{n}\right\| \\
& \quad \leq\left\|T_{n} y_{n}-T_{n} x_{n}\right\|+\left\|T_{n} x_{n}-x_{n}\right\| \\
& \quad \leq L\left\|y_{n}-x_{n}\right\|+\left\|T_{n} x_{n}-x_{n}\right\| \longrightarrow 0
\end{aligned}
$$

as $n \longrightarrow \infty$.

Thus, from (33) and (35) we have that

$$
\begin{aligned}
& \left\|x_{n+1}-x_{n}\right\| \\
& =\| \alpha_{n}\left(f\left(x_{n}\right)-x_{n}\right)+\left(1-\alpha_{n}\right) \\
& \quad \times\left(a_{n} x_{n}+b_{n} T_{n} y_{n}+c_{n} G x_{n}\right)-x_{n} \| \\
& \leq \alpha_{n}\left\|f\left(x_{n}\right)-x_{n}\right\|+\left(1-\alpha_{n}\right) b_{n}\left\|T_{n} y_{n}-x_{n}\right\| \\
& \quad+\left(1-\alpha_{n}\right) c_{n}\left\|G x_{n}-x_{n}\right\| \longrightarrow 0 \text { as } n \longrightarrow \infty .
\end{aligned}
$$


Therefore, $\left\|x_{n+j}-x_{n}\right\| \rightarrow 0$, as $n \rightarrow \infty$, for all $j=$ $1,2, \ldots, M$, and hence

$$
\begin{aligned}
& \left\|x_{n}-T_{n+j} x_{n}\right\| \\
& \leq\left\|x_{n}-x_{n+j}\right\|+\left\|x_{n+j}-T_{n+j} x_{n+j}\right\| \\
& \quad+L\left\|x_{n+j}-x_{n}\right\| \longrightarrow 0,
\end{aligned}
$$

as $n \rightarrow \infty$, for all $j \in\{1,2, \ldots, M\}$.

Now, since $\left\{x_{n}\right\}$ is bounded subset of $H$, we can choose a subsequence $\left\{x_{n_{m}}\right\}$ of $\left\{x_{n}\right\}$ such that $x_{n_{m}} \rightarrow x$ and $\limsup _{n \rightarrow \infty}\left\langle f\left(x^{*}\right)^{m}-x^{*}, x_{n}-x^{*}\right\rangle=\lim _{m \rightarrow \infty}\left\langle f\left(x^{*}\right)-\right.$ $\left.x^{*}, x_{n_{m}}-x^{*}\right\rangle$. Then, from (37) and Lemma 6 we have that $x \in F\left(T_{j}\right)$, for each $j=1,2, \ldots, M$. Hence, $x \in \cap_{j=1}^{M} F\left(T_{j}\right)$.

In addition, since $G$ is nonexpansive, from Lemma 6 we get that $x \in F(G)$ and hence by Lemmas 4 and 3 we obtain that $x \in \operatorname{VI}\left(C, A_{j}\right)$, for each $j \in\{1,2, \ldots, N\}$.

Therefore, by Lemma 5, we immediately obtain that

$$
\begin{aligned}
& \limsup _{n \rightarrow \infty}\left\langle f\left(x^{*}\right)-x^{*}, x_{n}-x^{*}\right\rangle \\
& \quad=\lim _{m \rightarrow \infty}\left\langle f\left(x^{*}\right)-x^{*}, x_{n_{m}}-x^{*}\right\rangle \\
& =\left\langle f\left(x^{*}\right)-x^{*}, x-x^{*}\right\rangle \leq 0 .
\end{aligned}
$$

Then, it follows from (32), (38), and Lemma 9 that $\| x_{n}-$ $x^{*} \| \rightarrow 0$ as $n \rightarrow \infty$. Consequently, $x_{n} \rightarrow x^{*}=P_{\mathscr{F}}\left(f\left(x^{*}\right)\right)$.

Case 2. Suppose that there exists a subsequence $\left\{n_{i}\right\}$ of $\{n\}$ such that

$$
\left\|x_{n_{i}}-x^{*}\right\|<\left\|x_{n_{i}+1}-x^{*}\right\|
$$

for all $i \in \mathbb{N}$. Then, by Lemma 8 , there exists a nondecreasing sequence $\left\{m_{k}\right\} \subset \mathbb{N}$ such that $m_{k} \rightarrow \infty$, and

$$
\left\|x_{m_{k}}-x^{*}\right\| \leq\left\|x_{m_{k}+1}-x^{*}\right\|, \quad\left\|x_{k}-x^{*}\right\| \leq\left\|x_{m_{k}+1}-x^{*}\right\|,
$$

for all $k \in \mathbb{N}$. Now, from (29) and (23) we get that $x_{m_{k}}-$ $T_{m_{k}} x_{m_{k}} \rightarrow 0$ and $G x_{n_{k}}-x_{n_{k}} \rightarrow 0$ as $k \rightarrow \infty$. Thus, following the method in Case 1, we obtain that $x_{m_{k}+1}-x_{m_{k}} \rightarrow$ $0, x_{m_{k}}-T_{j} x_{m_{k}} \rightarrow 0$, and

$$
\limsup _{k \rightarrow \infty}\left\langle f\left(x^{*}\right)-x^{*}, x_{m_{k}}-x^{*}\right\rangle \leq 0 .
$$

Furthermore, from (32) and (40) we obtain that

$$
\begin{aligned}
& \alpha_{m_{k}}(1-2 \alpha)\left\|x_{m_{k}}-x^{*}\right\|^{2} \\
& \leq\left\|x_{m_{k}}-x^{*}\right\|^{2}-\left\|x_{m_{k}+1}-x^{*}\right\|^{2} \\
&+2 \alpha_{m_{k}}\left\langle f\left(x^{*}\right)-x^{*}, x_{m_{k}}-x^{*}\right\rangle \\
&+2 \alpha_{m_{k}}\left\|x_{m_{k}+1}-x_{m_{k}}\right\|\left\|f\left(x_{m_{k}}\right)-x^{*}\right\| \\
& \leq 2 \alpha_{m_{k}}\left\langle f\left(x^{*}\right)-x^{*}, x_{m_{k}}-x^{*}\right\rangle \\
&+2 \alpha_{m_{k}}\left\|x_{m_{k}+1}-x_{m_{k}}\right\|\left\|f\left(x_{m_{k}}\right)-x^{*}\right\| .
\end{aligned}
$$

Now, using the fact that $\alpha_{m_{k}}>0$ and (41) we get that

$$
\left\|x_{m_{k}}-x^{*}\right\|^{2} \longrightarrow 0 \text { as } k \longrightarrow \infty,
$$

and this together with (32) implies that $\left\|x_{m_{k}+1}-x^{*}\right\| \rightarrow$ 0 as $k \rightarrow \infty$. Since $\left\|x_{k}-x^{*}\right\| \leq\left\|x_{m_{k}+1}-x^{*}\right\|$ for all $k \in \mathbb{N}$, we obtain that $x_{k} \rightarrow x^{*}$. Hence, from the above two cases, we can conclude that $\left\{x_{n}\right\}$ converges strongly to a point $x^{*}=P_{\mathscr{F}} f\left(x^{*}\right)$, which satisfies the variational inequality $\left\langle(I-f)\left(x^{*}\right), x-x^{*}\right\rangle \geq 0$, for all $x \in \mathscr{F}$. The proof is complete.

If, in Theorem 10, we assume that $f(x)=u \in C$, a constant mapping, then we get the following corollary.

Corollary 11. Let $C$ be a nonempty, closed, and convex subset of a real Hilbert space $H$. Let $T_{j}: C \rightarrow C, j=1,2, \ldots, M$, be Lipschitz pseudocontractive mappings with Lipschitz constants $L_{j}$, respectively. Let $A_{j}: C \rightarrow H$, for $j=1,2, \ldots, N$, be $\gamma_{j}$-inverse strongly accretive mappings. Assume that $\mathscr{F}=$ $\left[\cap_{j=1}^{M} F\left(T_{j}\right)\right] \bigcap\left[\cap_{j=1}^{N} \operatorname{VI}\left(C, A_{j}\right)\right]$ is nonempty. Let a sequence $\left\{x_{n}\right\}$ be generated from an arbitrary $x_{0}, u \in C$ by

$$
\begin{gathered}
y_{n}=\left(1-\lambda_{n}\right) x_{n}+\lambda_{n} T_{n} x_{n} ; \\
x_{n+1}=\alpha_{n} u+\left(1-\alpha_{n}\right)\left(a_{n} x_{n}+b_{n} T_{n} y_{n}+c_{n} G x_{n}\right),
\end{gathered}
$$

where $T_{n}=T_{n(\bmod M)}, G:=e_{0} I+e_{1} P_{C}\left[I-\gamma A_{1}\right]+e_{2} P_{C}\left[I-\gamma A_{2}\right]+$ $\cdots+e_{N} P_{C}\left[I-\gamma A_{r}\right]$, for $\gamma \in\left(0,2 \gamma_{0}\right)$, for $\gamma_{0}:=\min _{1 \leq j \leq N}\left\{\gamma_{j}\right\}$ with $e_{0}+e_{1}+\cdots+e_{r}=1$, and $b_{n}+c_{n} \leq \lambda_{n} \leq \lambda<1 /\left(\sqrt{1+L^{2}}+\right.$ $1), \forall n \geq 0$, for $L=\max \left\{L_{j}: 1 \leq j \leq M\right\}$. Then, $\left\{x_{n}\right\}$ converges strongly to a unique point $x^{*} \in C$ satisfying $x^{*}=P_{\mathscr{F}}(u)$, which is the unique solution of the variational inequality $\left\langle x^{*}-u, x-\right.$ $\left.x^{*}\right\rangle \geq 0$ for all $x \in \mathscr{F}$.

If, in Theorem 10, we assume that $N=1$ and $M=1$, then we get the following corollary which is Theorem 3.1 of [21].

Corollary 12. Let $C$ be a nonempty, closed, and convex subset of a real Hilbert space $H$. Let $T: C \rightarrow C$ be Lipschitz pseudocontractive mappings with Lipschitz constant $L$ and $A$ : $C \rightarrow H$ an $\gamma$-inverse strongly accretive mapping. Let $f$ : $C \rightarrow C$ be a contraction with constant $\alpha$. Assume that $\mathscr{F}=$ $F(T) \bigcap V I(C, A)$ is nonempty. Let a sequence $\left\{x_{n}\right\}$ be generated from an arbitrary $x_{0} \in C$ by

$$
\begin{aligned}
y_{n}= & \left(1-\lambda_{n}\right) x_{n}+\lambda_{n} T x_{n} ; \\
x_{n+1}= & \alpha_{n} f\left(x_{n}\right) \\
& +\left(1-\alpha_{n}\right)\left(a_{n} x_{n}+b_{n} T y_{n}+c_{n} P_{C}[I-r A] x_{n}\right),
\end{aligned}
$$

where $\in(0,2 \gamma)$ and $b_{n}+c_{n} \leq \lambda_{n} \leq \lambda<1 /\left(\sqrt{1+L^{2}}+1\right), \forall n \geq$ 0 . Then, $\left\{x_{n}\right\}$ converges strongly to a point $x^{*} \in \mathscr{F}$, which is the unique solution of the variational inequality $\left\langle(I-f)\left(x^{*}\right), x-\right.$ $\left.x^{*}\right\rangle \geq 0$ for all $x \in \mathscr{F}$.

If, in Theorem 10, we assume that $T_{i}^{\prime}$ s are strictly pseudocontractive mappins, then we get the following corollary. 
Corollary 13. Let $C$ be a nonempty, closed, and convex subset of a real Hilbert space $H$. Let $T_{i}: C \rightarrow C, i=1,2, \ldots M$, be $\lambda_{i^{-}}$ strictly pseudocontractive mappings and let $A_{i}: C \rightarrow H$, for $i=1,2, \ldots, N$, be an $\gamma_{i}$-inverse strongly accretive mappings. Let $f: C \rightarrow C$ be a contraction with constant $\alpha$. Assume that $\mathscr{F}=\left[\cap_{i=1}^{M} F\left(T_{i}\right)\right] \bigcap\left[\cap_{i=1}^{N} V I\left(C, A_{i}\right)\right]$ is nonempty. Let a sequence $\left\{x_{n}\right\}$ be generated from an arbitrary $x_{0} \in C$ by

$$
\begin{aligned}
y_{n}= & \left(1-\lambda_{n}\right) x_{n}+\lambda_{n} T_{n} x_{n} ; \\
x_{n+1}= & \alpha_{n} f\left(x_{n}\right) \\
& +\left(1-\alpha_{n}\right)\left(a_{n} x_{n}+b_{n} T_{n} y_{n}+c_{n} G x_{n}\right),
\end{aligned}
$$

where $T_{n}=T_{n(\bmod M)}, G:=c_{0} I+e_{1} P_{C}\left[I-\gamma A_{1}\right]+e_{2} P_{C}\left[I-\gamma A_{2}\right]+$ $\cdots+e_{N} P_{C}\left[I-\gamma A_{r}\right]$, for $\gamma \in\left(0,2 \gamma_{0}\right)$, for $\gamma_{0}:=\min _{1 \leq i \leq N}\left\{\gamma_{i}\right\}$ with $e_{0}+e_{1}+\cdots+e_{r}=1$, and $b_{n}+c_{n} \leq \lambda_{n} \leq \lambda<$ $1 /\left(\sqrt{1+L^{2}}+1\right), \forall n \geq 0, L=\max \left\{\left(1+\lambda_{i}\right) / \lambda_{i}\right\}$. Then, $\left\{x_{n}\right\}$ converges strongly to a point $x^{*} \in \mathscr{F}$, which is the unique solution of the variational inequality $\left\langle(I-f)\left(x^{*}\right), x-x^{*}\right\rangle \geq 0$ for all $x \in \mathscr{F}$.

If, in Theorem 10, we assume that $T_{i}^{\prime}$ s are nonexpansive mapping, then we get the following corollary.

Corollary 14. Let $C$ be a nonempty, closed, and convex subset of a real Hilbert space $H$. Let $T_{i}: C \rightarrow C, i=1,2, \ldots, M$, be nonexpansive mappings and let $A_{i}: C \rightarrow H$, for $i=$ $1,2, \ldots, N$, be an $\gamma_{i}$-inverse strongly accretive mappings. Let $f: C \rightarrow C$ be a contraction with constant $\alpha$. Assume that $\mathscr{F}=$ $\left[\cap_{i=1}^{M} F\left(T_{i}\right)\right] \bigcap\left[\cap_{i=1}^{N} \operatorname{VI}\left(C, A_{i}\right)\right]$ is nonempty. Let a sequence $\left\{x_{n}\right\}$ be generated from an arbitrary $x_{0} \in C$ by

$$
\begin{aligned}
y_{n}= & \left(1-\lambda_{n}\right) x_{n}+\lambda_{n} T_{n} x_{n} ; \\
x_{n+1}= & \alpha_{n} f\left(x_{n}\right) \\
& +\left(1-\alpha_{n}\right)\left(a_{n} x_{n}+b_{n} T_{n} y_{n}+c_{n} G x_{n}\right),
\end{aligned}
$$

where $T_{n}=T_{n(\bmod M)}, G=c_{0} I+e_{1} P_{C}\left[I-\gamma A_{1}\right]+e_{2} P_{C}\left[I-\gamma A_{2}\right]+$ $\cdots+e_{N} P_{C}\left[I-\gamma A_{r}\right]$, for $\gamma \in\left(0,2 \gamma_{0}\right)$, for $\gamma_{0}:=\min _{1 \leq i \leq N}\left\{\gamma_{i}\right\}$ with $e_{0}+e_{1}+\cdots+e_{r}=1, a_{n}+b_{n}+c_{n}=1$, and $b_{n}+c_{n} \leq$ $\lambda_{n} \leq \lambda<1 /(\sqrt{2}+1), \forall n \geq 0$. Then, $\left\{x_{n}\right\}$ converges strongly to point $x^{*} \in \mathscr{F}$, which is the unique solution of the variational inequality $\left\langle(I-f)\left(x^{*}\right), x-x^{*}\right\rangle \geq 0$ for all $x \in \mathscr{F}$.

We note that the method of proof of Theorem 10 provides the following theorem which is a convergence theorem for a minimum norm point of common fixed points of a finite family of Lipschitz pseudocontractive mappings and common solutions of a finite family of variational inequality problems for accretive mappings.

Theorem 15. Let $C$ be a nonempty, closed, and convex subset of a real Hilbert space $H$. Let $T_{j}: C \rightarrow C, j=1,2, \ldots, M$, be Lipschitz pseudocontractive mappings with Lipschitz constants $L_{j}$, respectively. Let $A_{j}: C \rightarrow H$, for $j=1,2, \ldots, N$, be $\gamma_{j}$-inverse strongly accretive mappings. Assume that $\mathscr{F}=$
$\left[\cap_{j=1}^{M} F\left(T_{j}\right)\right] \bigcap\left[\cap_{j=1}^{N} \operatorname{VI}\left(C, A_{j}\right)\right]$ is nonempty. Let a sequence $\left\{x_{n}\right\}$ be generated from an arbitrary $x_{0} \in C$ by

$$
\begin{aligned}
y_{n} & =\left(1-\lambda_{n}\right) x_{n}+\lambda_{n} T_{n} x_{n} ; \\
x_{n+1} & =P_{C}\left[\left(1-\alpha_{n}\right)\left(a_{n} x_{n}+b_{n} T_{n} y_{n}+c_{n} G x_{n}\right)\right],
\end{aligned}
$$

where $T_{n}=T_{n(\bmod M)}, G:=e_{0} I+e_{1} P_{C}\left[I-\gamma A_{1}\right]+e_{2} P_{C}\left[I-\gamma A_{2}\right]+$ $\cdots+e_{N} P_{C}\left[I-\gamma A_{r}\right]$, for $\gamma \in\left(0,2 \gamma_{0}\right)$, for $\gamma_{0}:=\min _{1 \leq j \leq N}\left\{\gamma_{j}\right\}$ with $e_{0}+e_{1}+\cdots+e_{r}=1$, and $b_{n}+c_{n} \leq \lambda_{n} \leq \lambda<$ $1 /\left(\sqrt{1+L^{2}}+1\right), \forall n \geq 0$, for $L=\max \left\{L_{j}: 1 \leq j \leq M\right\}$. Then, $\left\{x_{n}\right\}$ converges strongly to a unique minimum norm point $x^{*}$ of $\mathscr{F}$ (i.e., $x^{*}=P_{\mathscr{F}}(0)$ ), which is the unique solution of the variational inequality $\left\langle x^{*}, x-x^{*}\right\rangle \geq 0$ for all $x \in \mathscr{F}$.

\section{Numerical Example}

Now, we give an example of two Lipschitz pseudocontractive mappings and two $\gamma$-inverse strongly accretive mappings satisfying Theorem 10 and some numerical experiment result to explain the conclusion of the theorem as follows.

Example 1. Let $H=\mathbb{R}$ with absolute value norm. Let $C=$ $[-2,2]$ and let $T_{1}, T_{2}: C \rightarrow C$ be defined by

$$
\begin{gathered}
T_{1} x:= \begin{cases}x+x^{2}, & x \in[-2,0], \\
x, & x \in(0,2],\end{cases} \\
T_{2} x:= \begin{cases}x, & x \in\left[-2, \frac{1}{2}\right], \\
x-\left(\frac{16}{9}\right)\left(x-\frac{1}{2}\right)^{2}, & x \in\left(\frac{1}{2}, 2\right] .\end{cases}
\end{gathered}
$$

Clearly, for $x, y \in C$ we have that

$$
\begin{aligned}
& \left\langle\left(I-T_{1}\right) x-\left(I-T_{1}\right) y, x-y\right\rangle \geq 0, \\
& \left\langle\left(I-T_{2}\right) x-\left(I-T_{2}\right) y, x-y\right\rangle \geq 0
\end{aligned}
$$

which show that both mappings are pseudocontractive. Next, we show that $T_{1}$ is Lipschitz with $L=5$. If $x, y \in[-2,0]$, then

$$
\begin{aligned}
\left|T_{1} x-T_{1} y\right| & =\left|x+x^{2}-y-y^{2}\right| \\
& =|(x+y)+1||x-y| \leq 3|x-y| .
\end{aligned}
$$

If $x, y \in(0,2]$, then

$$
\left|T_{1} x-T_{1} y\right|=|x-y| .
$$

If $x \in[-2,0]$ and $y \in(0,2]$, then

$$
\begin{aligned}
& \left|T_{1} x-T_{1} y\right| \\
& \quad=\left|x+x^{2}-y\right| \\
& \quad=\left|x-y+x^{2}\right|=\left|x-y+x^{2}-y^{2}+y^{2}\right| \\
& =\left|x-y+x^{2}-y^{2}\right|+y^{2} \\
& \quad \leq|x+y+1| \cdot|x-y|+|y-x|^{2} \\
& \quad=(|x+y+1|+|x+y|) \cdot|x-y| \leq 5|x-y| .
\end{aligned}
$$


Thus, we get that $T_{1}$ is Lipschitz pseudocontractive with $L=5$ and $F\left(T_{1}\right)=[0,2]$ which is not nonexpansive, since if we take $x=-2$ and $y=-1.9$, we have that $\left|T_{1} x-T_{2} y\right|=$ $0.29>0.1=|x-y|$. Similarly, we can show that $T_{2}$ is Lipschitz pseudocontractive with $L=4$ and $F\left(T_{2}\right)=[-2,1 / 2]$ which is not nonexpansive.

Furthermore, for $C=[-2,2]$, let $A_{1}, A_{2}: C \rightarrow \mathbb{R}$ be defined by

$$
\begin{aligned}
& A_{1} x:= \begin{cases}-\left(x-\frac{1}{2}\right)^{2}, & x \in\left[-2, \frac{1}{2}\right), \\
0, & x \in\left[\frac{1}{2}, 2\right],\end{cases} \\
& A_{2} x:= \begin{cases}0, & x \in\left[-2, \frac{2}{3}\right], \\
3\left(x-\frac{2}{3}\right)^{2}, & x \in\left(\frac{2}{3}, 2\right] .\end{cases}
\end{aligned}
$$

Then we first show that $A_{1}$ is $\gamma$-inverse strongly accretive mapping with $\gamma=1 / 5$.

If $x, y \in[-2,1 / 2)$, then

$$
\begin{aligned}
\left\langle A_{1} x-A_{1} y, x-y\right\rangle & =\left\langle-\left(x-\frac{1}{2}\right)^{2}+\left(y-\frac{1}{2}\right)^{2}, x-y\right\rangle \\
& =\left[\left(x-\frac{1}{2}\right)^{2}-\left(y-\frac{1}{2}\right)^{2}\right](y-x) \\
& =\left[\left(x-\frac{1}{2}\right)^{2}-\left(y-\frac{1}{2}\right)^{2}\right]\left[\left(y-\frac{1}{2}\right)-\left(x-\frac{1}{2}\right)\right] \\
& =\left[\left(x-\frac{1}{2}\right)^{2}-\left(y-\frac{1}{2}\right)^{2}\right] \frac{\left[(y-1 / 2)^{2}-(x-1 / 2)^{2}\right]}{(y-1 / 2)+(x-1 / 2)} \\
& =\left[\left(x-\frac{1}{2}\right)^{2}-\left(y-\frac{1}{2}\right)^{2}\right] \frac{\left[(x-1 / 2)^{2}-(y-1 / 2)^{2}\right]}{(1 / 2-x)+(1 / 2-y)} \\
\geq & \frac{1}{5}\left|\left(x-\frac{1}{2}\right)^{2}-\left(y-\frac{1}{2}\right)^{2}\right|^{2} \\
= & \frac{1}{5}\left|A_{1} x-A_{1} y\right|^{2} .
\end{aligned}
$$

If $x \in[-2,1 / 2)$ and $y \in[1 / 2,2]$, we get that

$$
\begin{aligned}
& \left\langle A_{1} x-A_{1} y, x-y\right\rangle \\
& =\left\langle-\left(x-\frac{1}{2}\right)^{2}, x-y\right\rangle=\left(x-\frac{1}{2}\right)^{2}(y-x) \\
& =\left(x-\frac{1}{2}\right)^{2}\left[\left(y-\frac{1}{2}\right)-\left(x-\frac{1}{2}\right)\right] \\
& \geq\left(x-\frac{1}{2}\right)^{2}\left(\frac{1}{2}-x\right) \\
& =\left(x-\frac{1}{2}\right)^{2} \frac{(1 / 2-x)^{2}}{(1 / 2-x)} \geq \frac{2}{5}\left|\left(x-\frac{1}{2}\right)^{2}\right|^{2} \\
& \geq \frac{1}{5}\left|A_{1} x-A_{1} y\right|^{2} .
\end{aligned}
$$

TABLE 1

\begin{tabular}{lccc}
\hline$u=0.6$ & $x_{0}=1$ & $u=0.8$ & $x_{0}=-1$ \\
$n$ & $x_{n}$ & $n$ & $x_{n}$ \\
\hline 0 & 1.0000 & 0 & -1.0000 \\
500 & 0.6112 & 5000 & 0.0627 \\
10,000 & 0.5137 & 10,000 & 0.4282 \\
12,000 & 0.5121 & 15,000 & 0.4540 \\
14,000 & 0.5110 & 20,000 & 0.4686 \\
18,000 & 0.5093 & 25,000 & 0.4782 \\
20,000 & 0.5087 & 35,000 & 0.4905 \\
\hline
\end{tabular}
hence

If $x, y \in[1 / 2,2]$, then we get that $\left|A_{1} x-A_{1} y\right|=0$ and

$$
\left\langle A_{1} x-A_{1} y, x-y\right\rangle \geq \frac{1}{5}\left|A_{1} x-A_{1} y\right|^{2} .
$$

Therefore, $A_{1}$ is $\gamma$-inverse strongly accretive mapping with $\gamma=1 / 5$ and $\operatorname{VI}\left(C, A_{1}\right)=[1 / 2,2]$. Similarly, we can show that $A_{2}$ is $\gamma$-inverse strongly accretive mapping with $\gamma=1 / 2$ and $\operatorname{VI}\left(C, A_{2}\right)=[-2,2 / 3]$. $\{1 / 2\}$.

Note that we have $F\left(T_{1}\right) \cap F\left(T_{2}\right) \cap \operatorname{VI}\left(C, A_{1}\right) \cap \operatorname{VI}\left(C, A_{2}\right)=$

Thus, taking $\alpha_{n}=1 /(10 n+100), \lambda_{n}=2 /(n+100)+$ $0.065, b_{n}=c_{n}=1 /(n+100)+0.01, a_{n}=1-2 /(n+$ $100)-0.02$, and $f(x)=u \in C$, we observe that conditions of Theorem 10 are satisfied and Scheme (17) provides the following Table 1 and Figures 1(a) and 1(b) for $u=0.6$ and $u=0.8$, respectively.

We observe that the data provides strong convergence of the sequence to the common point of fixed points of both pseudocontractive mappings and solutions of both variational inequality problems for $\gamma$-inverse strongly accretive mappings.

Remark 2. Theorem 10 provides an iteration scheme which converges strongly to a common point of fixed points of a finite family of Lipschitzian pseudocontractive mappings and solutions of a finite family of variational inequality problems in Hilbert spaces.

Remark 3. Theorem 10 improves Theorem 3.1 of Takahashi and Toyoda [19], Iiduka and Takahashi [20], and Zegeye and Shahzad [21] and Theorem 3.2 of Yao et al. [22] in the sense that our convergence is to a common point of fixed points of a finite family of Lipschitzian pseudocontractive mappings and solutions of a finite family of variational inequality problems.

\section{Conflict of Interests}

The authors declare that they have no conflict of interests regarding the publication of this paper.

\section{Acknowledgments}

This project was funded by the Deanship of Scientific Research (DSR), King Abdulaziz University, Jeddah, under grant no. 167/130/1434. The authors, therefore, acknowledge 


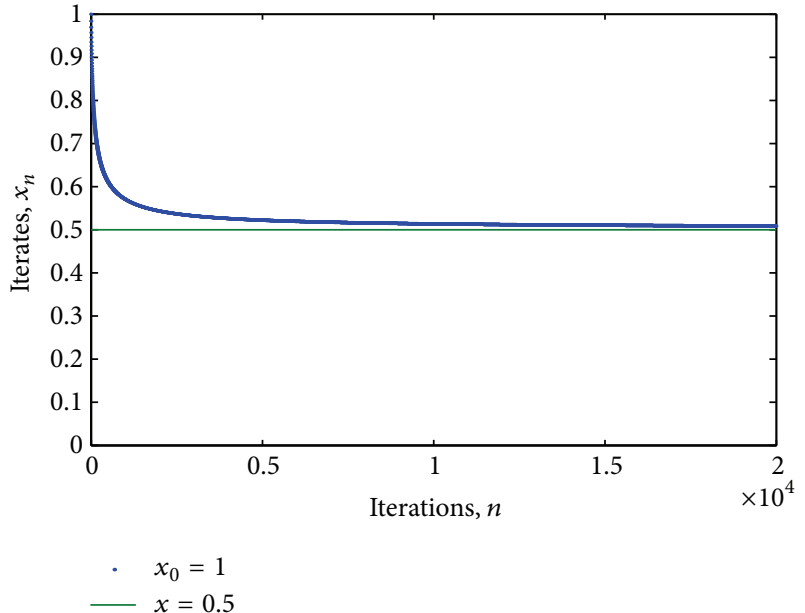

(a)

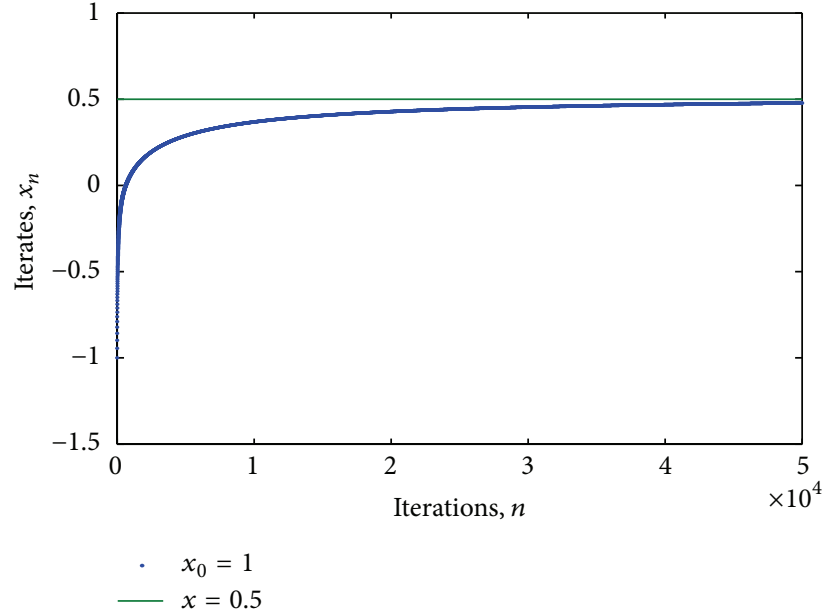

(b)

Figure 1

with thanks DSR technical and financial support. The authors also thank the referees for their valuable comments and suggestions, which improved the presentation of this paper.

\section{References}

[1] D. Kinderlehrer and G. Stampaccia, An Iteration to Variational Inequalities and Their Applications, Academic Press, New York, NY, USA, 1990.

[2] H. Zegeye and N. Shahzad, "A hybrid scheme for finite families of equilibrium, variational inequality and fixed point problems," Nonlinear Analysis: Theory, Methods \& Applications, vol. 74, no. 1, pp. 263-272, 2011.

[3] H. Zegeye and N. Shahzad, "Approximating common solution of variational inequality problems for two monotone mappings in Banach spaces," Optimization Letters, vol. 5, no. 4, pp. 691704, 2011

[4] H. Zegeye and N. Shahzad, "Strong convergence theorems for variational inequality problems and quasi- $\phi$-asymptotically nonexpansive mappings," Journal of Global Optimization, vol. 54, no. 1, pp. 101-116, 2012.

[5] H. Zegeye and N. Shahzad, "An iteration to a common point of solution of variational inequality and fixed point-problems in Banach spaces," Journal of Applied Mathematics, vol. 2012, Article ID 504503, 19 pages, 2012.

[6] F. E. Browder and W. V. Petryshyn, "Construction of fixed points of nonlinear mappings in Hilbert space," Journal of Mathematical Analysis and Applications, vol. 20, pp. 197-228, 1967.

[7] L. C. Ceng, A. Petruşel, and J. C. Yao, "Composite viscosity approximation methods for equilibrium problem, variational inequality and common fixed points," Journal of Nonlinear and Convex Analysis, vol. 15, no. 2, pp. 219-240, 2014.

[8] L. C. Ceng, A. Petrusel, M. M. Wong, and J. C. Yao, "Hybrid algorithms for solving variational inequalities, variational inclusions, mixed equilibria, and fixed point problems," Abstract and Applied Analysis, vol. 2014, Article ID 208717, 22 pages, 2014.
[9] L. Ceng, A. Petrusel, and M. Wong, "Strong convergence theorem for a generalized equilibrium problem and a pseudocontractive mapping in a Hilbert space," Taiwanese Journal of Mathematics, vol. 14, no. 5, pp. 1881-1901, 2010.

[10] D. R. Sahu, V. Colao, and G. Marino, "Strong convergence theorems for approximating common fixed points of families of nonexpansive mappings and applications," Journal of Global Optimization, vol. 56, no. 4, pp. 1631-1651, 2013.

[11] D. R. Sahu and A. Petruşel, "Strong convergence of iterative methods by strictly pseudocontractive mappings in Banach spaces," Nonlinear Analysis: Theory, Methods \& Applications, vol. 74, no. 17, pp. 6012-6023, 2011.

[12] Y. Yao, Y. Liou, and N. Shahzad, "Construction of iterative methods for variational inequality and fixed point problems," Numerical Functional Analysis and Optimization, vol. 33, no. 10, pp. 1250-1267, 2012.

[13] Y. Yao, G. Marino, and L. Muglia, "A modified Korpelevich's method convergent to the minimum-norm solution of a variational inequality," Optimization, vol. 63, no. 4, pp. 559-569, 2014.

[14] Y. Yao and M. Postolache, "Iterative methods for pseudomonotone variational inequalities and fixed-point problems," Journal of Optimization Theory and Applications, vol. 155, no. 1, pp. 273$287,2012$.

[15] H. Zegeye, E. U. Ofoedu, and N. Shahzad, "Convergence theorems for equilibrium problem, variational inequality problem and countably infinite relatively quasi-nonexpansive mappings," Applied Mathematics and Computation, vol. 216, no. 12, pp. 3439-3449, 2010.

[16] H. Zegeye and N. Shahzad, "A hybrid approximation method for equilibrium, variational inequality and fixed point problems," Nonlinear Analysis: Hybrid Systems, vol. 4, no. 4, pp. 619-630, 2010.

[17] H. Zegeye and N. Shahzad, "Strong convergence theorems for a solution of finite families of equilibrium and variational inequality problems," Optimization, vol. 63, no. 2, pp. 207-223, 2014.

[18] H. Zegeye and N. Shahzad, "Extragradient method for solutions of variational inequality problems in Banach spaces," Abstract and Applied Analysis, vol. 2013, Article ID 832548, 8 pages, 2013. 
[19] W. Takahashi and M. Toyoda, "Weak convergence theorems for nonexpansive mappings and monotone mappings," Journal of Optimization Theory and Applications, vol. 118, no. 2, pp. 417428, 2003.

[20] H. Iiduka and W. Takahashi, "Strong convergence theorems for nonexpansive mappings and inverse-strongly monotone mappings," Nonlinear Analysis: Theory, Methods \& Applications, vol. 61, no. 3, pp. 341-350, 2005.

[21] H. Zegeye and N. Shahzad, "Solutions of variational inequality problems in the set of fixed points of pseudocontractive mappings," Carpathian Journal of Mathematics, vol. 30, no. 2, pp. 257-265, 2014.

[22] Y. Yao, Y. C. Liou, and S. M. Kang, "Algorithms construction for variational inequalities," Fixed Point Theory and Applications, vol. 2011, Article ID 794203, 12 pages, 2011.

[23] Y. Cai, Y. Tang, and L. Liu, "Iterative algorithms for minimumnorm fixed point of non-expansive mapping in Hilbert space," Fixed Point Theory and Applications, vol. 2012, article 49, 2012.

[24] K. Aoyama, H. Iiduka, and W. Takahashi, "Weak convergence of an iterative sequence for accretive operators in Banach spaces," Fixed Point Theory and Applications, vol. 2006, Article ID 35390, 13 pages, 2006.

[25] C. E. Chidume, H. Zegeye, and E. Prempeh, "Strong convergence theorems for a finite family of nonexpansive mappings in Banach spaces," Communications on Applied Nonlinear Analysis, vol. 11, no. 2, pp. 25-32, 2004.

[26] Y. I. Alber, "Metric and generalized projection operators in Banach spaces: properties and applications," in Theory and Applications of Nonlinear Operators of Accretive and Monotone Type, Lecture Notes in Pure and Applied Mathematics, pp. 1550, Marcel Dekker, New York, NY, USA, 1996.

[27] Q. B. Zhang and C. Z. Cheng, "Strong convergence theorem for a family of Lipschitz pseudocontractive mappings in a Hilbert space," Mathematical and Computer Modelling, vol. 48, no. 3-4, pp. 480-485, 2008.

[28] H. Zegeye and N. Shahzad, "Convergence of Mann's type iteration method for generalized asymptotically nonexpansive mappings," Computers and Mathematics with Applications, vol. 62, no. 11, pp. 4007-4014, 2011.

[29] P. E. Maingé, "Strong convergence of projected subgradient methods for nonsmooth and nonstrictly convex minimization," Set-Valued Analysis, vol. 16, no. 7-8, pp. 899-912, 2008.

[30] H. K. Xu, "Another control condition in an iterative method for nonexpansive mappings," Bulletin of the Australian Mathematical Society, vol. 65, no. 1, pp. 109-113, 2002. 


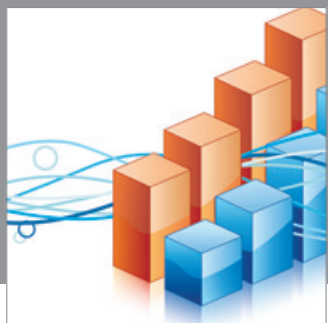

Advances in

Operations Research

mansans

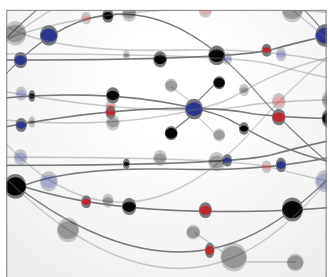

The Scientific World Journal
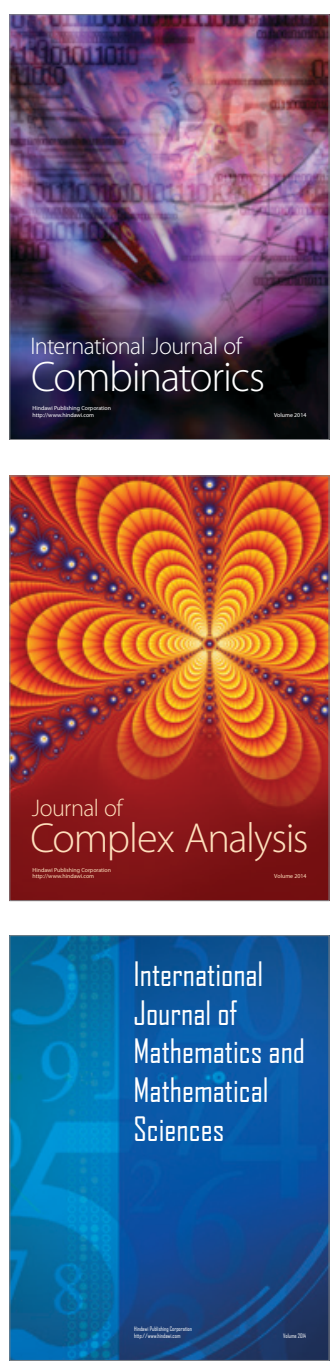
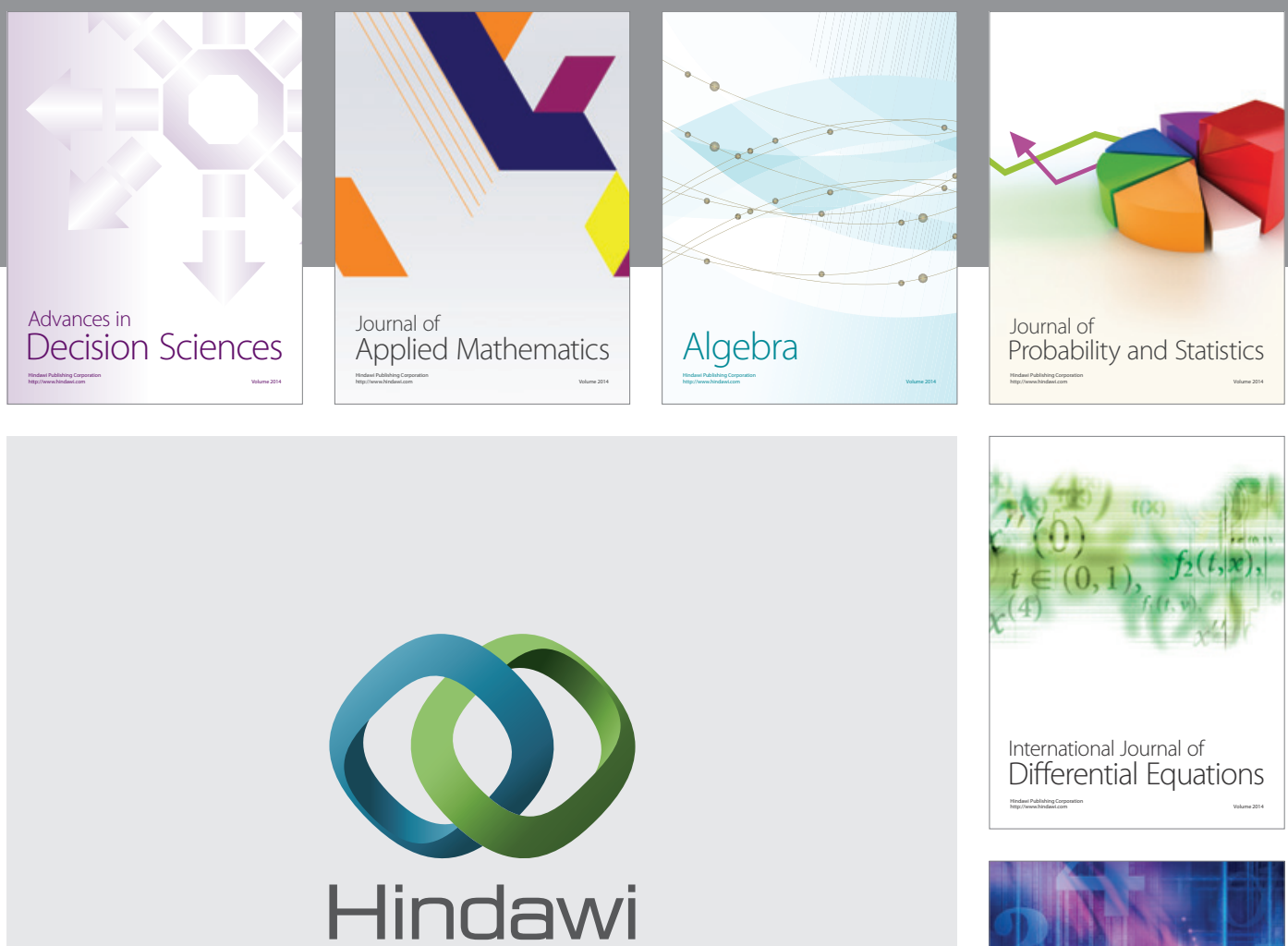

Submit your manuscripts at http://www.hindawi.com
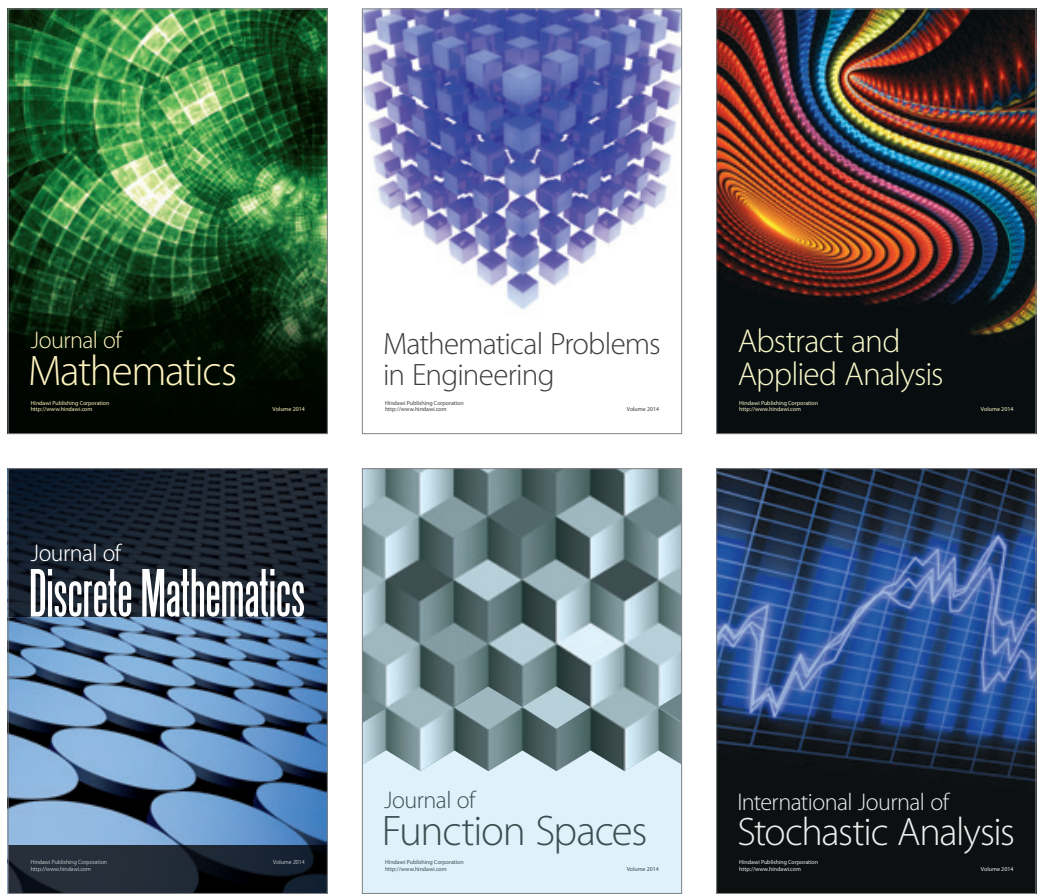

Journal of

Function Spaces

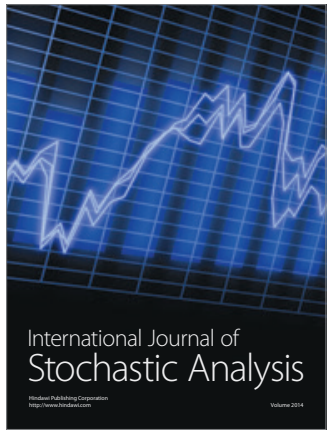

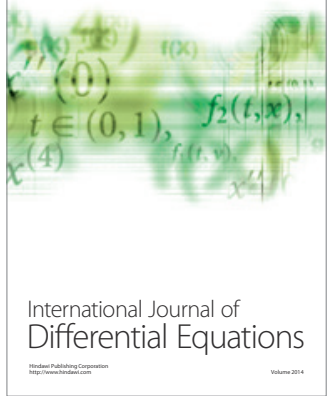
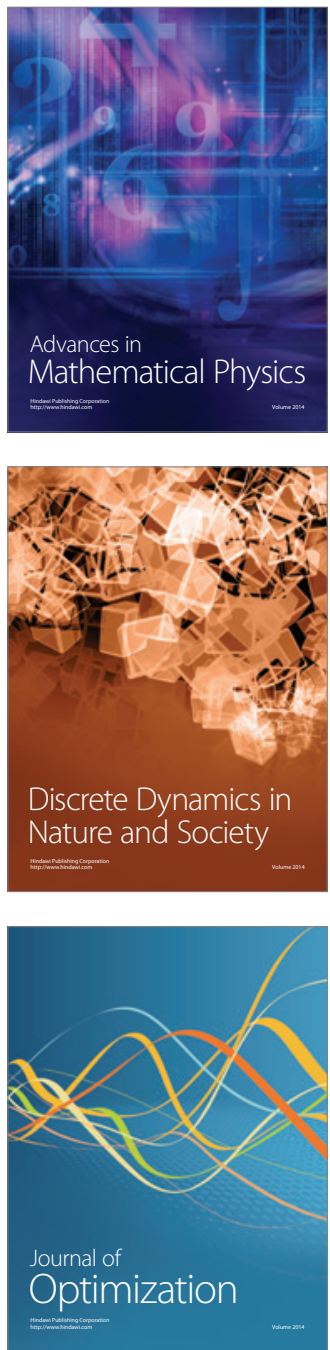